\title{
Low-resolution spectrograph for the IOTA interferometer
}

Sam Ragland, Wesley A. Traub, Rafael Millan-Gabet, Nathaniel P. Carleton, Ettore Pedretti

Sam Ragland, Wesley A. Traub, Rafael Millan-Gabet, Nathaniel P. Carleton, Ettore Pedretti, "Low-resolution spectrograph for the IOTA interferometer," Proc. SPIE 4838, Interferometry for Optical Astronomy II, (21 February 2003); doi: $10.1117 / 12.459321$

Event: Astronomical Telescopes and Instrumentation, 2002, Waikoloa, Hawai'i, United States 


\title{
A Low-resolution Spectrograph for the IOTA interferometer
}

\author{
${ }^{1}$ S. Ragland ${ }^{\mathrm{a}, *}$, W.A.Traub ${ }^{\mathrm{a}}$, R. Millan-Gabet ${ }^{\mathrm{a},{ }^{*}}$, N.P. Carleton ${ }^{\mathrm{a}}$, and E. Pedretti ${ }^{\text {abc,*** }}$ \\ ${ }^{a}$ Harvard-Smithsonian Center for Astrophysics, Cambridge, Massachusetts \\ ${ }^{\mathrm{b}}$ College de France, Paris, France \\ ${ }^{c}$ Observatoire de Haute Provence, France
}

\begin{abstract}
The design and scientific objectives of a near infrared channeled spectrometer planned at the IOTA interferometer are discussed. The spectrometer has the flexibility to reconfigure easily for conventional broadband operations in addition to multi-channel mode. This instrument makes use of the existing PICNIC camera at the IOTA in order to be cost efficient. The spectrometer has been designed specifically for studying Mira stars. However, it will find its application in other areas of astrophysical interests such as studies of circumstellar disks around young stars and binary stars.
\end{abstract}

\section{INTRODUCTION}

Mira variables are large-amplitude, long-period, pulsating cool giants. Their importance for the studies of stellar evolution results from their position at the tip of the asymptotic giant branch of the HR diagram and their high mass loss rate.

Mira stars have been subject to extensive studies during last decade using various high-angularresolution techniques such as lunar occultation, speckle interferometry, aperture masking and long-baseline interferometry in the visible and infrared wavelengths (Haniff, Scholz \& Tuthill, 1995; Tuthill, Haniff \& Baldwin, 1995; van Belle et al. 1996; Burns et al., 1998; Perrin et al., 1999; van Belle et al., 1999; Tej et al., 1999; Tuthill et al., 2000; Weiner et al., 2000; Young et al., 2000; Hofmann et al., 2001; Thompson, 2002). These measurements have provided physical parameters such as radius and temperature of these cool stars. However, little is known about the structures and the dynamics of their extended cool atmospheres and their role in stellar pulsation and mass loss. The mode of pulsation is still an unresolved problem. The cool atmospheres of these evolved stars are a breeding ground for complex molecules and dust grains, modulating the spectra of the underlying continuum-forming region with absorption and emission features. While, these atmospheric signatures are useful in studying atmospheric structures, opacities and shock wave characteristics, they restrict our capabilities to probe down to the continuumforming region without any contamination from the cooler atmospheric layers. Accurate monochromatic size measurements in the continuum are very crucial to validate Mira model atmospheres (Jacob et al., 2000; Scholz, 2001; Scholz, 2002). Unfortunately, the present generation of interferometers suffers sensitivity limitations apart from calibration issues. Hence, visibility measurements are made mostly in standard broad bands, and in narrow bands at shorter wavelengths partly to avoid fringe smearing. There are no homogeneous sets of measurements available in the literature that are taken simultaneously at different wavelengths as a function of Mira phase in the continuum without molecular contamination. Moreover, most size estimations are based on visibility measurements taken at a single or a few closely spaced spatial frequencies resulting in biased size estimations for Mira-like stars with extended atmospheres. We have begun a systematic program of observing long period variables at IOTA with all three telescopes using a recently incorporated PICNIC detector. We measure closure phase in addition to visibility amplitude, which is crucial for detecting asymmetries in the brightness profile of these cool stars and to some extent for measuring limb darkening as well.

\footnotetext{
${ }^{1}$ Send correspondence to S. Ragland: sragland@cfa.harvard.edu

* Michelson postdoctoral fellows

** Smithsonian predoctoral fellow
} 
While near-infrared measurements provide near-continuum size measurements, more accurate recent visibility data show departures from Mira model curves. In addition, large size changes as a function of wavelength and phase have been reported. Although molecular contamination can not be ruled out as a possible reason for these observed effects, photospheric asymmetries or surface features or circumstellar emissions could very well be the reason. These effects are not taken into account in any Mira model work. Resolving Mira atmospheres spatially and spectrally in the continuum and molecular bands with photometric phase in the near infrared region would enable us to understand better opacity sources in Mira atmosphere, and to investigate the role of pulsation and shock waves in molecule and dust formation. The opacity in the stellar atmosphere changes when the size and hence the temperature changes. The channeled spectrometer presented here is a new capability planned at the IOTA in the $1-2.5 \mu \mathrm{m}$ spectral region, which would improve our capability to address unresolved problems in the area of Mira atmospheres. The spectral resolution of this instrument is a compromise between the sensitivity, and the capability to resolve the continuum and to some extent molecular bands. These measurements could provide vital input to understand the atmospheric structures of this interesting class of objects.

\section{INSTRUMENT DESIGN}

The design objective is to develop a channeled spectrometer without much change to the existing setup at the IOTA (Traub, 2002), utilizing existing hardware maximally. The spectrometer has been designed to work with beams from all three telescopes, enabling spectrally resolved visibility and phase-closure measurements in the continuum and molecular bands of the atmosphere of evolved stars at near infrared wavelengths. The six interferometric outputs of the three-telescope configuration are spectrally dispersed using a prism and re-imaged onto the PICNIC camera.

\subsection{Optical Design:}

We used the ZEMAX Optical Design Program to design the optics for this channeled spectrometer. The design goal was to have about $90 \%$ of the light in one pixel of the PICNIC array for each channel in the 12.5 micron region. There are two types of beam-combiners available at the IOTA for three-beam combination, namely, the IONIC/PICNIC beam-combiner (Berger et al., 2002) which is an integrated optics based unit, and the classical free-space beam-combiner which is near completion. Single mode fibers are used for spatial filtering in both cases at some or other stage - before beam combination in the case of the IONIC/PICNIC beam-combiner and after the beam combination in the case of the free-space beamcombiner. The ends of the six output fibers/planar wave guides are placed along the vertical axis separated by about $250 \mu \mathrm{m}$. The beams from them are collimated, dispersed in the horizontal axis and then focused onto the PICNIC array, which is cooled to $\mathrm{LN}_{2}$ temperature. The beam-combiners and the dispersive optics are at room temperature. This configuration enables us (a) to reconfigure easily the system for multichannel and broadband filter modes of operations, (b) optimize the optics for the science camera, and (c) easily change the spectral resolution depending on the science requirements.

The optical schematic of the spectrometer is shown in Figure 1. The system consists of six lenses and one dispersive element. These lenses form two sets of triplets, each with two BaF2 and one Infrasil lenses. The details of the triplets are given in Table 1. All lens surfaces are spherical and broadband antireflection coated. The optical system was optimized using ZEMAX, and the resulting spot diagram is shown in Figure 2. The through-focus spot diagram is shown in Figure 3. The design presented here is based on presently available test plates. Although several prism, grism and grating based designs over a wide range of spectral resolutions have been worked out, due to sensitivity considerations, a single element $\mathrm{CaF}_{2}$ prism with a prism angle of $36^{\circ}$ has been planned for the initial version of this spectrometer. Since the dispersive optics is placed outside the PICNIC dewar, its easy to change it during an observing night.

Our initial attempt was to design a doublet for collimating and re-imaging. We came out with a design based on $\mathrm{CaF}_{2}$-IRG7 doublet optics, which was compact and had a good spot diagram, but later we found that IRG7 glasses are presently not manufactured (Oliva \& Gennari, 1998). After several attempts with many infrared glasses, we realized the need to go for triplet optics for broadband applications. We designed the collimating and re-imaging optics to be identical in order to reduce the cost. We also tried an 
off-axis mirror design, but it was relatively hard to meet the field of view requirements in order to accommodate all six interferometric beams.

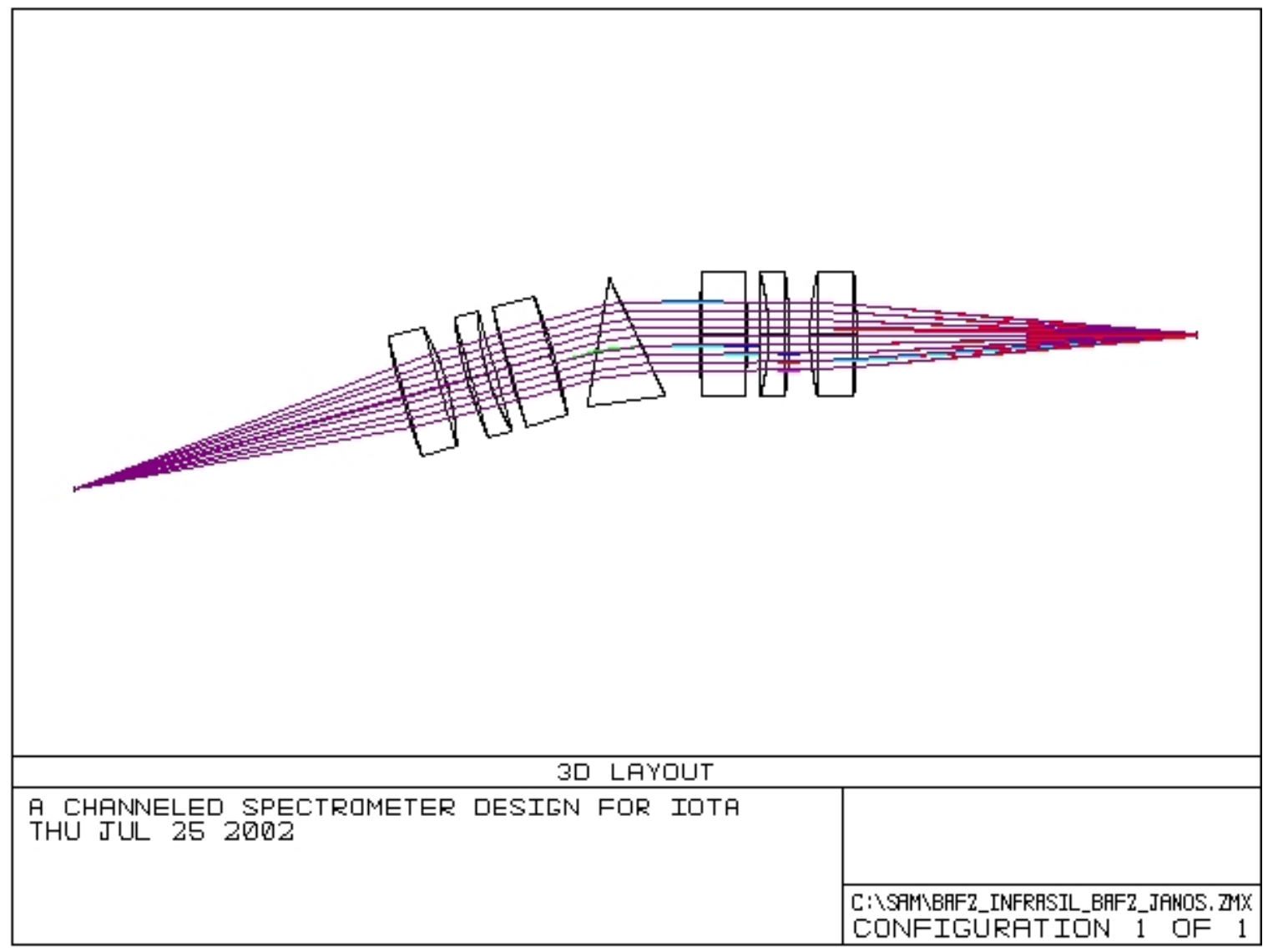

Figure 1: The optical layout of the channeled near infrared spectrometer. The design is symmetric, but in this diagram the input is on the left, and output on the right. All 6 interferometric inputs are placed along the z-axis (normal to the paper). The 6 output spectra are likewise arrayed along 6 levels of the z-axis, and dispersed in the y-axis (vertical direction). The dispersed beams are too close together to be visually distinguishable in this diagram.

The background emissions in the $\mathrm{J}$ and $\mathrm{H}$ bands will not limit the performance of this spectrometer. However in K-band, and even in the short K'-band, we do experience background at IOTA. The present design minimizes this effect in two ways. (a) The beam is single-mode, so the only background radiation is that which is within the star beam's single electromagnetic mode (due to emissivity of the optics train), and this cannot be removed by any mechanism (except with lower-emissivity optics). In particular, light from outside this mode will not be detected, to the extent that the single mode is well-matched in area and solid angle to the detector pixel. This mode-matching between the fiber output and the detector pixel is an advantage of the proposed system. (b) The beam is spectrally dispersed, so any background radiation in the single mode will also be dispersed, reducing the load on the detector pixel, and allowing for a less-rapid discharge of the PICNIC pixel, which is an advantage in observing bright stars. 
Table 1: The surface data of the triplet optics

\begin{tabular}{|c|r|c|l|}
\hline $\begin{array}{c}\text { Surface } \\
\text { Number }\end{array}$ & $\begin{array}{c}\text { Radius of } \\
\text { Curvature (mm) }\end{array}$ & $\begin{array}{c}\text { Distance to next } \\
\text { element (mm) }\end{array}$ & Glass \\
\hline Object & 218.20 & 110.0 & \\
$\mathbf{1}$ & -84.463 & 15.0 & BaF2 \\
$\mathbf{2}$ & 142.831 & 6.7 & \\
$\mathbf{3}$ & 75.630 & 6.7 & Infrasil \\
$\mathbf{4}$ & 371.120 & 15.3 & BaF2 \\
$\mathbf{5}$ & -202.761 & & \\
$\mathbf{6}$ & & & \\
\hline
\end{tabular}

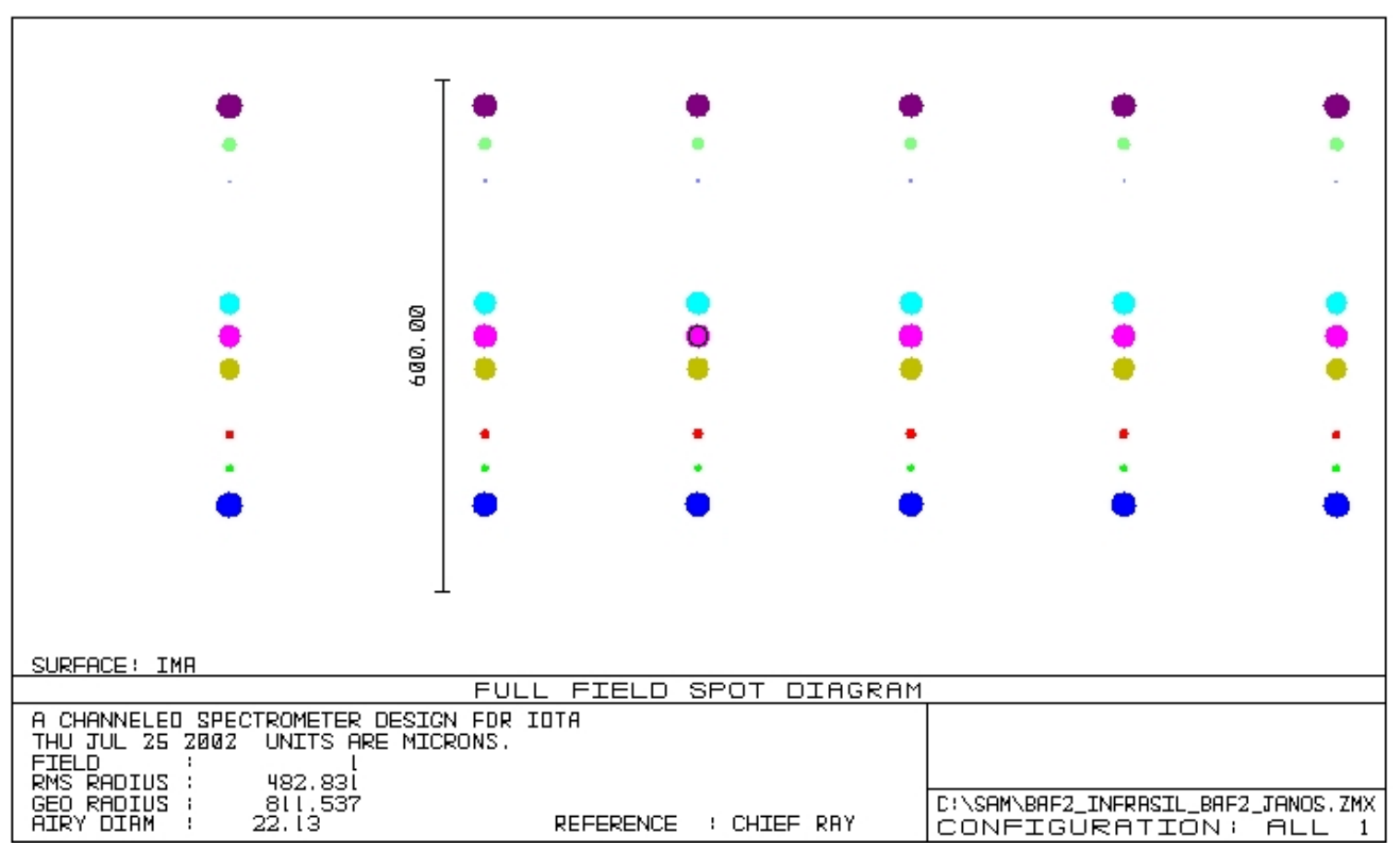

Figure 2: Full-field spot diagram of the optics. Only the central wavelengths of the three channels centered in the $\mathrm{J}, \mathrm{H}$ and $\mathrm{K}$ bands are shown here for clarity. The six columns correspond to six interferometric beams and the nine rows correspond to nine channels out of 14 possible channels. The wavelength increases as we go up. Each spot is approximately centered in a $40 \mu \mathrm{m}$ detector pixel. The collimating and camera lenses will be adjusted slightly in magnification to match the $250 \mu \mathrm{m}$ fiber spacing onto $240 \mu \mathrm{m}$ (6column) centers. 


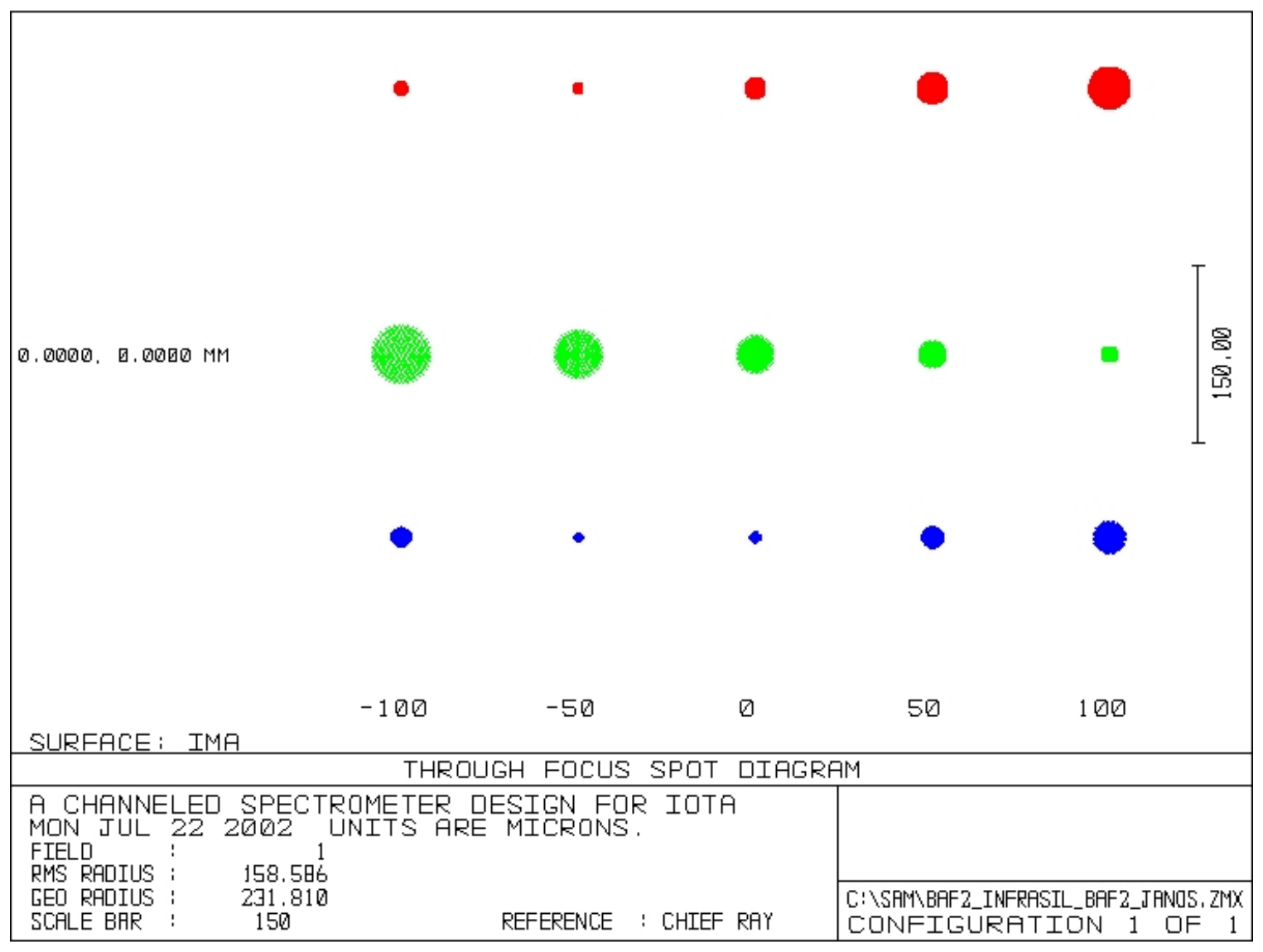

Figure 3: Through-focus spot diagram is shown here. The three rows correspond to central wavelengths of J, H \& K bands (bottom to top). The columns show the spot diagram around the focal plane (in and out of focus) in steps of $50 \mu \mathrm{m}$.

\subsection{Mechanical Design:}

The dispersive element is mounted on a circular platform containing two projected arms that can rotate with respect to the circular platform. The collimating and camera optics are mounted one each on these projected arms. The collimating arm, the circular platform and the beam-combiner outputs are fixed while the imaging arm and the PICNIC dewar are adjusted either for channeled spectrometer mode or for broadband filter mode for which the dispersive element is removed. Alternatively, the PICNIC camera and the imaging arm could be fixed, while input beams and the collimating arm are adjusted.

\subsection{Electronics:}

The data acquisition and control system of this spectrometer is based on the ALTERA Complex Programmable Logic Device (CPLD) technology described in a paper by Pedretti et al. (2002) in this SPIE proceeding. The reconfigurability of this component enables us easily to change the electronics of the spectrometer from channeled spectrometer mode to broadband filter mode in a matter of a few milliseconds. 
Different readout modes have been worked out in order to optimize the usage of this spectrometer for different observing programs and different observing conditions.

- Full spectral range reading within the coherence time: This mode involves reading 14 X $6=84$ pixels (and skipping several pixels in between) in $15-60 \mathrm{~ms}$ depending on the seeing condition. The ADC card presently in use at the IOTA is not meant for such a fast mode. We will acquire a fast ADC for this mode of operation.

- Reading one spectral band at a time: This mode involves reading all the five spectral channels of a given spectral band within the coherence time. Essentially, J, H \& K regions are read separately, one after another. A frame of 5 X $6=30$ pixels is read within the coherence time. This readout mode is not useful for wavelength bootstrapping operations described below. However, this mode of operation is essential to observe moderately faint sources or bright sources under worst seeing conditions. This mode can be implemented with the existing ADC card at IOTA.

- Reading only the central spectral channel of the $J, H \& K$ bands within the coherence time: This readout mode will be used in the case of very faint sources or targets for which the interest is only in the continuum wavelengths. A group of $3 \times 6=18$ pixels is read within the coherence time. In this mode of operation, the pixels are read several times within the coherence time in order to increase the signal-to-noise ratio.

\section{WAVELENGTH \& BASELINE BOOTSTRAPPING}

Simultaneous recording of interference fringes in several channels in the JHK bands would enable us to develop a more robust fringe tracker system at the IOTA which makes use of the science data itself when the OPD scanning rate is adequately fast. For the purpose of fringe tracking, the dispersed channel outputs are averaged when it is required. Presently, we have adopted baseline bootstrapping (Mozurkewich \& Armstrong, 1992) whereby fringes are tracked in two short baselines, while science data are recorded in all three baselines simultaneously enabling low visibility closure-phase measurements.

In addition, wavelength-bootstrapping operations (Mozurkewich, 1999) will be implemented with this spectrometer - while tracking fringes in the $\mathrm{K}$ band channels, low visibility measurements will be made in the $\mathrm{J} \& \mathrm{H}$ bands. This mode of operation is very crucial to achieve some of our scientific objectives discussed here.

It is possible that continuum channels in the $\mathrm{J}, \mathrm{H} \& \mathrm{~K}$ bands resolve the same spatial extent in some special cases such as binary star observations. In such cases, each continuum channel provides visibility measurement at a different spatial frequency and hence better $\mathrm{u}, \mathrm{v}$ coverage for imaging studies.

\section{SCIENCE DRIVER}

The scientific objectives are (a) to obtain accurate continuum size estimations, (b) to measure limb darkening, and (c) to detect asymmetry in the stellar disc (if any) from near infrared visibility and closurephase measurements. The high spatial frequencies are essential to look for signatures of limb darkening and possible small scale surface features, and the low and medium spatial frequencies are essential to resolve the contributions from a possible circumstellar (gas/dust) envelope and emission from upper atmospheric layers of Mira stars. In addition, size measurements in the molecular bands are useful to study the atmospheric structures in detail, although it is very hard to make these measurements without contamination from underlying strong continuum forming regions. Moreover, available Mira models don't accurately account for molecular opacities. Our molecular band observations would provide a basis and a simulation for improving Mira models.

Our observational strategy is to obtain wavelength and phase dependent visibility (and phaseclosure) measurements at as large a number of spatial frequencies as possible at the same epoch. Although reconstructing the stellar intensity profile from these measurements is presently an impossible task, these measurements would provide valuable input to the theoretical efforts in building more realistic Mira atmospheric models. Our primary interest is to obtain accurate continuum measurements that can be easily 
compared with the existing Mira models. These measurements could help improve the models and our understanding of Mira atmospheres, and help us to interpret the data taken at molecular absorption bands.

The source of dominant molecular opacity in the near infrared spectra of oxygen rich Miras is $\mathrm{H}_{2} \mathrm{O}$. In addition, bands of $\mathrm{CO}, \mathrm{OH}, \mathrm{TiO} \& \mathrm{VO}$ have been identified in the Mira spectra (Lançon \& RoccaVolmerange, 1992; Joyce et al., 1998; Lançon \& Wood, 2000; Alvarez et al., 2000). Absorption features of $\mathrm{CN}$ are dominant in carbon rich atmospheres in this wavelength range. In addition, $\mathrm{C}_{2} \&$ weak features of $\mathrm{CO}$ are seen in their spectra apart from several unidentified absorption features (Loidl, Lançon \& Jørgensen, 2001). S star spectra contain absorption features of molecules such as TiS, ZrS, TiO, and CN. The central channels of $\mathrm{J}, \mathrm{H} \& \mathrm{~K}$ bands would provide near-continuum size measurements and the wings would provide a measure of molecular upper layers such as $\mathrm{H}_{2} \mathrm{O}, \mathrm{CO}, \mathrm{VO} \& \mathrm{TiO}$ in oxygen-rich atmospheres. It is worth mentioning here that the $\mathrm{H}$ continuum is not free from $\mathrm{CO} \& \mathrm{OH}$ molecular contamination in oxygen rich Miras. Similarly, $\mathrm{J}$ continuum contains features of TiO \& VO molecules and their strength depends on the Mira phase. In the case of carbon stars, $\mathrm{CN} \& \mathrm{C}_{2}$ molecular zones can be probed with this spectrometer. It is hard to find a continuum channel for $\mathrm{S}$ stars because of crowded molecular features. We would like to address the following questions:

- What are the sources of opacity in Mira atmospheres? How complete a picture can we form of the Mira atmosphere by measuring low spectral resolution visibilities in the $1-2.5 \mu \mathrm{m}$ region?

- How does the molecular opacity change with photometric phase?

- Is there any phase lag between the continuum and molecular band cycles? How does this relate to the shock wave propagation in the cool atmosphere?

- What are the roles of stellar pulsation and shock waves in molecules and dust formation?

- What about the mode of pulsation in Mira stars?

Departure from circular symmetry has been known in long-period variables from various high angular resolution observations (Karovska et al., 1991; Haniff et al., 1992; Tuthill, Haniff \& Baldwin, 1999; Monnier, Tuthill \& Danchi, 2000). However, the origin of the distortion is still not clear; the observed asymmetries are interpreted either in terms of elliptical distortions or an otherwise circular symmetric photosphere containing asymmetric compact features. It is also important to determine whether asymmetries are common in Mira-like stars, since the presence of substantial asymmetries could have strong impact on the derived stellar parameters and the model atmospheres of Mira stars. A systematic study of the morphology of Mira-like stars with pulsation phase has been undertaken at the IOTA interferometer in order to characterize the observed photospheric asymmetries and surface features. The time-evolution of the asymmetry is again a subject of uncertainty. The evolution time scale is a useful diagnostic tool in understanding the physics of the observed asymmetry. The phase-closure observing capability of IOTA is vital for these studies.

\section{ACKNOWLEDGEMENTS}

This work was performed in part under contract with the Jet Propulsion Laboratory (JPL) through the Michelson Fellowship program funded by NASA as an element of the planet finder program. JPL is managed for NASA by the California Institute of Technology.

\section{REFERENCES}

1. Alvarez, R., Lançon, A., Plez, B., Wood, P. R., "Optical and near-infrared spectrophotometric properties of Long Period Variables and other luminous red stars", A\&A, 353, 322, 2000.

2. Burns, D., Baldwin, J. E., Boysen, R. C., Haniff, C. A., Lawson, P. R. et al., "Large-amplitude periodic variations in the angular diameter of $R$ Leonis", MNRAS, 297, 462, 1998.

3. Berger, J.P. et al., "First Results from the IONIC Three Beam Combiner at IOTA", Interferometry for Optical Astronomy II, Traub, W.A., Ed., Proc. SPIE 4838, this volume, 2002. 
4. Haniff, C. A., Scholz, M., and Tuthill, P. G., "New Diameter Measurements of 10 Mira Variables Implications for Effective Temperatures Atmospheric Structure and Pulsation Modes", MNRAS, 276, 640, 1995.

5. Haniff, C. A., Ghez, A. M., Gorham, P. W., Kulkarni, S. R., Matthews et al., "Optical aperture synthetic images of the photosphere and molecular atmosphere of Mira", AJ, 103, 1662, 1992.

6. Hofmann, K.-H., Balega, Y., Scholz, M., Weigelt, G., "Multi-wavelength bispectrum speckle interferometry of R Leo and comparison with Mira star models", A\&A, 376, 518, 2001.

7. Jacob, A. P., Bedding, T. R., Robertson, J. G., Scholz, M., "Wavelength dependence of angular diameters of $M$ giants: an observational perspective", MNRAS, 312, 733, 2000.

8. Joyce, R.R.;, Hinkle, K.H., Wallace, L., Dulick, M., Lambert, D.L., "Spectra of Cool Stars in the J Band (1.0-1.3 $\mu ; m)$ at Medium Resolution", AJ, 116, 2520, 1998.

9. Karovska, M., Nisenson, P., Papaliolios, C., Boyle, R.P., "Asymmetries in the atmosphere of Mira", ApJ, 374, L51, 1991.

10. Lançon, A., Rocca-Volmerange, B., "A library of near-IR stellar spectra from 1.328 to 2.5 microns" A\&AS, 96, 593, 1992.

11. Lançon, A., Wood, P. R.,"A library of 0.5 to $2.5 \mu \mathrm{m}$ spectra of luminous cool stars" A\&AS, 146, 217, 2000.

12. Loidl, R., Lançon, A., Jørgensen, U. G., "Spectra of carbon-rich asymptotic giant branch stars between 0.5 and $2.5 \mu \mathrm{m}$ : Theory meets observation”, A\&A, 371.1065, 2001.

13. Monnier, J. D.; Tuthill, P. G., Danchi, W. C., "Diffraction-limited Near-IR Imaging at Keck Reveals Asymmetric, Time-variable Nebula around Carbon Star CIT 6", ApJ, 545, 957, 2000.

14. Mozurkewich, D., "Interferometer Design for synthesis Imaging", in Principles of Long baseline Stellar Interferometry, Ed. Lawson, P.R., p.231, 1999.

15. Mozurkewich, D., Armstrong, J. T., "Array Layout and Beam Combination Considerations Necessary for Imaging with a Long Baseline Optical Interferometer" in High-Resolution Imaging by Interferometry II. Ground-Based Interferometry at Visible and Infrared Wavelengths, Beckers, J.M., Merkle, F., Eds., Proc. ESO Conference, 39, 801, 1992.

16. Oliva, E., \& Gennari, S. 1998, A\&AS, 128, 5890

17. Pedretti, E., Millan-Gabet, R., Monnier, J.D., Morel, S., Traub, W.A. et al., "Reconfigurable electronics at the IOTA interferometer", Interferometry for Optical Astronomy II, Traub, W.A., Ed., Proc. SPIE 4838, this volume, 2002.

18. Perrin, G., Coudé du Foresto, V., Ridgway, S. T., Mennesson, B., Ruilier, C. et al., "Interferometric observations of $R$ Leonis in the $K$ band. First direct detection of the photospheric pulsation and study of the atmospheric intensity distribution", A\&A, 345, 221, 1999.

19. Scholz, M., "On the interpretation of stellar disc observations in terms of diameters", MNRAS, 321, 347, 2001.

20. Scholz, M., "Mira Science with Interferometry: a review", Interferometry for Optical Astronomy II, Traub, W.A., Ed., Proc. SPIE 4838, this volume, 2002.

21. Tej, A., Chandrasekhar, T., Ashok, N. M., Ragland, S., Richichi, A. et al., "The Angular Diameter of the Mira Variable R Leonis at 3.36 and 2.2 Microns", AJ, 117, 1857, 1999.

22. Tuthill, P. G., Danchi, W. C.;, Hale, D. S., Monnier, J. D., Townes, C. H., "Near-and Mid-Infrared Subarcsecond Structure of the Dusty Symbiotic Star R Aquarii “, ApJ, 534., 907, 2000.

23. Tuthill, P. G., Haniff, C. A., Baldwin, J. E., "Surface imaging of long-period variable stars" , MNRAS, 306, 353, 1999.

24. Tuthill, P. G., Haniff, C. A., Baldwin, J. E., "Longterm Diameter Variations in the Longperiod Variable Omicron-Ceti", MNRAS, 277, 1541, 1995.

25. Traub, W.A., "Recent Progress at IOTA with 3 Telescopes", Interferometry for Optical Astronomy II, Traub, W.A., Ed., Proc. SPIE 4838, this volume, 2002.

26. Thompson, R.R., "Spectral angular diameters of Mira variables using long baseline near-infrared interferometry", PhD Thesis, University of Wyoming, 2002.

27. van Belle, G. T., Dyck, H. M., Benson, J. A., Lacasse, M. G., "Angular Size Measurements of 18 Mira Variable Stars at $2.2 \mu m "$, AJ, 112, 2147, 1996. 
28. van Belle, G. T., Lane, B. F., Thompson, R. R., Boden, A. F., Colavita, M. M. et al., "Radii and Effective Temperatures for G, K, and M Giants and Supergiants", AJ, 117, 521, 1999.

29. Weiner, J., Danchi, W. C., Hale, D. D. S., McMahon, J., Townes, C.H. et al.," Precision Measurements of the Diameters of $\alpha$ Orionis and o Ceti at 11 Microns", ApJ, 544, 1097, 2000.

30. Young, J. S., Baldwin, J. E., Boysen, R. C., Haniff, C. A., Pearson, D. et al., "Cyclic variations in the angular diameter of X Cyg“"., MNRAS, 318, 381, 2000. 